\title{
The digital memory game: an assistive technology resource evaluated by children with cerebral palsy
}

\author{
Sueli Neide Silva da Cunha ${ }^{1}$, Xisto Lucas Travassos Junior ${ }^{2}$, Renato Guizzo ${ }^{3}$ and Camila de Sousa Pereira-Guizzo ${ }^{* *}$
}

\begin{abstract}
The activities of daily living are routine self-maintenance tasks. Children with cerebral palsy can have difficulties, which can vary according to their level of motor impairment, in the performance of functional activities such as feeding and dressing themselves. The use of digital memory gaming can be a play activity and a technological resource that stimulates cognitive skills and contributes to the development of persons with disabilities. The objective of this article is to analyze the usability and effectiveness of a digital memory game for activities of daily living with children with cerebral palsy. Two children with cerebral palsy, one aged 5 and the other aged 7, participated in the investigation. The results obtained in the usability analysis are satisfactory because there were no major problems with the interface and the time available for the game and the rules presented were observed. The results also reveal the effectiveness of digital memory gaming for the recognition of objects and the activities of daily life. The importance of digital memory gaming for children with cerebral palsy is discussed.
\end{abstract}

Keywords: Activities of daily living, Digital gaming, Memory, Cerebral palsy, Assistive technology

\section{Background}

Cerebral palsy $(\mathrm{CP})$ is an injury to one or more parts of the brain that can occur during gestation, during delivery, or after birth. The causes may be varied: infectious diseases during pregnancy, maternal malnutrition, perinatal cerebral anoxia, and traumatic injury during birth, among other events. Motor disorder is CP's primary characteristic. These individuals may develop muscle weakness, difficulties controlling musculature, and changes in tonus (Beckung \& Hagberg, 2002; Mutch, Alberma, Hagber, Kodam, \& Perat, 1992). Other manifestations may be present, including changes in vision, speech disorders, and epilepsy. Thus, children with $\mathrm{CP}$ can exhibit certain difficulties in the performance of the everyday activities important for their functioning in different life settings.

Activities of daily living (ADL) are routine selfmaintenance tasks. These functional activities correspond to eating, dressing, hygiene, and communication habits (Guerzoni et al., 2008). According to the level of motor impairment, children with $\mathrm{CP}$ may have difficulties in the

\footnotetext{
* Correspondence: camila.pereira@fieb.org.br

${ }^{1}$ Faculdade de Tecnologia SENAI CIMATEC, Salvador, Brazil

Full list of author information is available at the end of the article
}

performance of ADL and lower social participation in household tasks (Brown \& Gordon, 1987). Promoting ADL performance has been the focus of interventions by many professionals and researchers. Interventions may be directed toward different objectives so that that the child acquires the greatest possible level of independence and autonomy in actions such as eating, taking a bath, performing personal hygiene, and dressing. From the perspectives of evidence-based practices, Guerzoni et al. (2008) conduct a systematic review of the literature, identifying the different types of interventions for the promotion of ADL in children with $\mathrm{CP}$, stressing the importance of connecting the health condition and the personal and environmental context factors in this process.

In the promotion of $\mathrm{ADL}$, although parental practices are variables that significantly influence functional development and the level of independence of children with $\mathrm{CP}$, the existence of different interventions and therapeutic processes is recognized. In short, interventions can currently rely on technological advancement or the employment of different technologies in learning, rehabilitation, and childhood development situations (Robertson \& Howells, 2008; Virvou \& Katsionis, 2008). The promotion of the functionality of persons with disabilities,

\section{Springer}

(c) 2016 Cunha et al. Open Access This article is distributed under the terms of the Creative Commons Attribution 4.0 International License (http://creativecommons.org/licenses/by/4.0/), which permits unrestricted use, distribution, and reproduction in any medium, provided you give appropriate credit to the original author(s) and the source, provide a link to the Creative Commons license, and indicate if changes were made. 
contextualized in scenarios of technological advances, may receive the contribution of assistive technology (United States, 2004).

In Brazilian legislation, assistive technology (AT) is an interdisciplinary area understood as the "products, resources, methods, strategies, practices, and services that seek to promote functionality related to the activity and participation of persons with disabilities, impairments, or reduced mobility, targeting their autonomy, independence, quality of life, and social inclusion" (Brazil, 2009, p.9). In this view of AT, among the many possibilities, digital gaming may be a resource that is capable of contributing to motor development, communication, and other cognitive processes of persons with disabilities.

Educational digital games explore play activities that have objectives directed toward development and learning. These games can have different types of challenges that, upon being overcome, stimulate several basic functions, such as attention, concentration, and memory (Connolly, Boyle, MacArthur, Hainey, \& Boyle, 2012). Additionally, Kearney (2005) finds that, in children with $\mathrm{CP}$, the skills learned in a game can be transferred to the real world and that such experiences can influence emotional and social development. Therefore, the application of games such as memory games may be an alternative for the development and learning of children with disabilities.

The traditional memory game is a well-known game that works on attention, concentration, and memorization and has many variations. The use of gaming to explore memory functions is of great relevance for the development of children because it is through their recollections that their individual experiences are constructed. Memories are typically classified according to duration, function, or content. According to function, memory can be classified as working memory, which has a duration ranging from seconds to minutes and serves to manage reality and process our acts. According to content, memories may be declarative, related to facts, events, or general knowledge; or procedural, which are memories of habits, motor skills, and sensory skills. According to duration, memories may be short-term, lasting from minutes to a few hours, the amount of time necessary for long-term memories to be consolidated (Izquierdo, 2011).

According to cognitive psychology, there are three common operations that represent a stage in the processing of memory. These operations are codification, storage, and recovery. According to Sternberg (2010), codification transforms sensory data into a form of mental representation, storage maintains the information codified in memory, and recovery withdraws or uses the stored information. With this process, it is possible to maintain and access information recorded in past experiences. Thus, a conceptual model related to the conception and application of the digital memory game tested in this study has been developed by the authors (Fig. 1).

The process in Fig. 1 first considers the directions given to children with $\mathrm{CP}$ by the mediator. The information addressed in the game is represented by symbols, which may be displayed as images and signs, interpreted by the child. The repetition of this process promotes the storage of information, which can be recovered at a later time, according to Sternberg (2010).

There are also other important elements that contribute to the construction of games and to the educational effects that they can promote. In the human-computer interface, an important evaluative aspect is usability. The International Organisation for Standardisation (1998) defines usability as the measure of a product, used by certain users, to achieve certain objectives with effectiveness (the analysis of the completion of the task and the quality of the result obtained), efficiency (the effort and resources necessary to arrive at the objective), and satisfaction (comfort and user acceptance) in a context of specific use. Thus, the usability analysis of an educational digital game can increase the assurance that the product will be simple, interesting, and satisfactory for the target audience.

Therefore, considering the importance of the conception and application of technological resources for the promotion of functionality and human development, this study aims to evaluate the usability of a digital memory game developed for children with CP and to analyze the effectiveness of this game for the association of corresponding objects and actions in ADL.

\section{Method}

\section{Study design}

The intervention was analyzed from a single-subject design perspective, which is conceived based on the need to define whether an experimental manipulation has had any effect on the individual participating in the investigation. This design treats the subjects individually in the decisions related to both the design itself and the processing of the collected data. In this study, the $A B$ design type was used, represented by "A" (baseline) and "B" (intervention) (Sampaio et al., 2008; Kazdin 1982; Lourenço, Hayashi, \& Almeida, 2009). The independent variable was the intervention, which consists of the application of the digital memory game to the child; the dependent variable was the child's performance on the association of pairs of cards with pictures of objects to the actions corresponding to ADL.

The study followed the precepts of Resolution 196/1996 of the Brazilian National Council of Health in relation to the Guidelines and Norms Regulating Research Involving Human Beings and was approved by the Research Ethics Committee of the Department of Health of the State of Bahia (368/2009). In addition to the institution's 


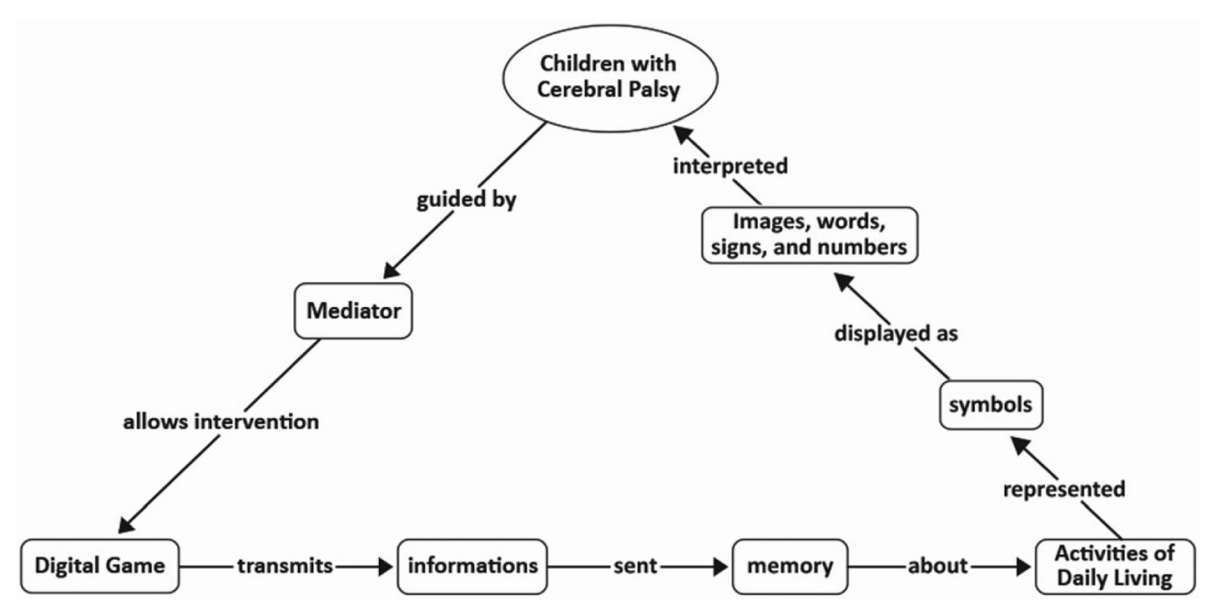

Fig. 1 Representation of the digital memory gaming concept in ADL. Source: Developed by the authors

authorization, the children's parents signed an informed consent form allowing the participation of the children in the present study.

\section{Participants}

The criteria for sample inclusion were as follows: children with $\mathrm{CP}$; being between the ages of 4 and 8 years old; being able to perform activities with the portable electronic equipment developed for the study; having some impairment in ADL; having the interest and consent to participate in the study.

Two children with $\mathrm{CP}$ participated in the study. For the analysis of game usability, the participant was a 7-year-old girl with cerebral palsy, speech disorder, motor coordination impairment, quadriplegic, and a dependent level in ADL according to the evaluation of Matsukura and Marturano (2001). For the analysis of the effectiveness of the digital game, the participant was a 5-year-old boy with cerebral palsy, speech disorder (dysarthria), motor coordination impairment, and a semi-independent level in ADL according to the evaluation of Matsukura and Marturano (2001).

\section{Site of data collection}

The study was performed at a public state institution that serves people with $\mathrm{CP}$ and other developmental disorders, located in the state of Bahia, Brazil. The study was conducted in the child-juvenile neuroevolutionary sector, which performs procedures with people with neurological dysfunction, such as $\mathrm{CP}$. The institution made adequate space and specialized assistance available for the study to be performed. In addition, during the game concept and development phase, some of the institution's professionals (a psychologist, an educational psychologist, and an occupational therapist) assisted in analyzing the usefulness of the proposal for the target population and the content addressed.

\section{Development of digital memory game for ADL}

Designed by the first author and developed by a specialized team, this game addresses the ADL theme, offering opportunities for children to use their cognitive skills, such as memory, for the resolution of the activity. The game is unique because it proposes an activity that involves the relationship between concepts of daily activities, allowing the child to learn by means of a play strategy. Special attention was given to the functionalities of the game, which were adapted to the needs of children with $\mathrm{CP}$. To provide for aspects that enable augmentative and alternative communication (AAC) and attention to the specific needs of the children, the game also uses pictures composed of a set of symbols called picture communication symbols (PCS), which were extracted from the "free communication board" of Project Amplisoft, free software for alternative communication (http:// www.ler.pucpr.br/amplisoft/).

The association of the proposed drawings - object versus action - addresses real aspects that relates to a person's everyday life. The ADL themes included the following: dressing, eating, and hygiene. The game was developed for one player and was composed of pairs of cards with complementary images. The card pairs were distributed randomly; thus, the player received the cards in different positions every time a game started. The game has four phases, with increasing levels of difficulty, and the phase the player is in, together with the option to leave the game, is always displayed on the screen. If the player selects an incorrect pair of cards, then the cards are highlighted in red, the move is undone, and the cards are returned to the previous position. If a correct pair is selected, then the cards are highlighted in green and disappear. When the player selects the last pair of the phase, Phase 1 of the game ends, and Phase 2 is displayed. This routine is performed in each of the phases until the end of Phase 4, the game's final stage. At the end of each phase, an image signaling the end 
of the phase and containing information on the time spent and the number of attempts is displayed.

Each phase of the game represents a daily activity that occurs at the start of the day (Phase 1), at lunchtime (Phase 2), in the afternoon (Phase 3) and at the end of the night (Phase 4). Phase 1 aims to develop daily activities that may occur at the start of the day. In this phase, the context is presented through the initial image of a child waking up. This image represents the situation of a daily morning routine, symbolized by the alarm clock and a landscape with the sun. Next, the phase starts with the display of six pairs of cards that correspond to possible morning activities, such as taking a bath, dressing, going to the bathroom, making the bed, brushing one's teeth, and combing one's hair. Upon clicking on the cards, the player must associate the pairs of actions with the corresponding objects, which are the toothbrush, the comb, the bed, the toilet paper, the shirt, and the shower. At the end, the phase closing image is displayed.

Phase 2 seeks to develop daily activities that can occur at the start of the afternoon, during lunchtime. The time of day is represented by a clock on the wall set to $12 \mathrm{pm}$ located beside a door. After this image, Phase 2 starts with the display of six pairs of cards, and the image of one card from each pair is shown while the other is hidden. The aim is to challenge the player a little more, increasing the level of difficulty. The player should first click on the card with the hidden image, which is revealed, and then identify and click on the card with the image corresponding to the card already revealed on the screen. In this phase, actions related to personal hygiene and the house are addressed, represented by an image of washed dishes, shoes being cleaned, feet wearing shoes, a person washing his/her hair, perfume being sprayed, and flossing one's teeth. Upon correctly associating these actions with the objects represented by the shampoo, the dental floss, the shoe brush, the sandal, the plate, and the deodorant, the phase is concluded.

Phase 3 takes place in the afternoon. On the side, there is a clock on the table set to $3 \mathrm{pm}$. After this image, the phase begins with the display of six pairs of cards, with the image in half of them revealed for a few seconds and then automatically hidden. Once again, the player is challenged with the increase in the game's level of difficulty. The player should take advantage of the time in which the cards are revealed to attempt to memorize them. The actions displayed in this phase correspond to routine activities, such as washing a fruit, washing a shirt, trimming one's toenails, blowing one's nose, drinking juice, and going to the dentist. These activities should be associated with an apple, a shirt, a foot, a tissue, a cup of juice, and a dentist, respectively. The player should join the pairs, clicking the two corresponding cards. The phase's final image is a child watching TV and eating an apple, having completed his/ her afternoon activities.

Phase 4 takes place at night. The last phase begins with the display of an image with the clock hanging on the wall set at $7 \mathrm{pm}$. After the display of the context image, the phase starts with the display of six pairs of cards, and the image on all cards is revealed for a few seconds and then automatically hidden. These cards correspond to daily activities such as drinking milk, moisturizing one's legs, cleaning one's ears, brushing one's teeth, washing one's hands, and putting on socks, represented by a carton of milk, a bottle of moisturizer, a cotton swab, toothpaste, a towel, and a sock, respectively. The player should click on one pair at a time until he or she is able to form all the correct pairs. At the end of the phase, the image of a child sleeping is displayed, symbolizing the conclusion of the daily routine.

The game presented is installed on a personal digital assistant (PDA). The language used is $\mathrm{C}++$ with a DirectFB graphics library. To play, pairs are formed, clicking directly on the apparatus's screen by means of a touchscreen display - a screen sensitive to touch that does not require use of a mouse or keyboard, allowing the player to interact using his/her finger or hand.

\section{Instruments \\ Rating Catalog of the Independence Level of Children from 4 to 8 years of age in Activities of Daily Living [Catálogo de Avaliação do Nível de Independência de Crianças de 4 a 8 anos nas Atividades de Vida Diária]}

This instrument, developed by Matsukura and Marturano (2001), evaluates 4 categories: eating, hygiene, dressing, and communication skills. In this catalog, there are five alternatives that consider: whether the child performs the task without physical or verbal assistance; whether the child performs with verbal assistance; whether the child performs with a demonstration; whether the child performs with physical assistance; or whether the child does not perform the task. The application of scoring criteria establishes the following levels: independent, semiindependent, and dependent.

\section{Rating record of picture hits}

Developed for this study, this instrument records children's hits associating objects with corresponding actions by means of pictures drawn on paper cards. An example of a record consists of an object (e.g., a ball) and an action (e.g., playing ball), with a space in front to assign a hit or miss in the association of the object with the action. The paper cards are composed of 10 pairs of pictures of objects and actions related to daily activities. The pictures of the objects used are a toothbrush, a plate of food, a bed, soap, a shirt, a tissue, a book, a ball, a glass of water, and pencils. The pictures used as ADL 
actions consist of a child doing the following: brushing his/her teeth, eating, waking up, bathing, putting on a shirt, blowing his/her nose, reading a book, cleaning his/ her ears, playing ball, drinking water, and drawing. The paper pictures were made based on the drawings from the picture communication symbols (PCS), a procedure also adopted in the illustrations of the digital game to maintain card standardization.

\section{Usability testing script}

This instrument was developed to evaluate the functionality of the game and the ease of use by the player in terms of: (a) game duration time; (b) the user's interaction with the interface (easy, regular, or difficult); (c) the understanding of the rules of the game (easy, regular, or difficult); (d) the correct association and recognition of the corresponding pictures (yes or no); (e) difficulty in differentiating the phases (yes or no); (f) consideration of the information made available: time, phase, and attempts during the game (yes or no); (g) gaming time in each phase; (h) the number of attempts in each phase; and (i) the level of difficulty encountered in each phase (low, medium, or high).

\section{Data collection and analysis}

The usability of the digital game was analyzed in a session lasting approximately one hour. The mediation was performed by the first author, with the aid of an occupational therapist. Directions for performing the usability test were given orally by the mediator. The occupational therapist contributed in positioning the child, considering the ergonomic conditions for the visualization of the game and performance in the execution of the task. The child well understood the instructions given, establishing communication primarily with the use of non-verbal components (visual contact, facial expression, and slight gesturing). Given that the child had difficulty in motor coordination, she pointed to the cards she considered to be correct. The mediator selected the command in the game, according to the child's indication. For the usability analysis, the researcher relied on two independent observers (trained professionals, one with experience in game scripting and the other in educational design). These observers independently evaluated the game's usability by means of the Usability Testing Script. The level of agreement between the observers was calculated as follows: agreement divided by the sum of agreement and disagreements, multiplied by 100 (Kazdin, 1982).

Regarding the experimental procedure, in the baseline phase, three sessions were performed with the child to ascertain the baseline's stability. The sessions were conducted on sequential days, in accordance with the participant's availability. These sessions were filmed to ensure a permanent record of the scenes and the child's performance. In this phase, there was no intervention on the part of the researcher in the choice of cards by the child, nor was reinforcement offered depending on the choice. To perform the evaluation, the pictures of ADL actions were spread out on a table, and the researcher displayed the pictures of the objects, one at a time, and asked the child to show the image that represented the use of the referenced object. The baseline evaluation was measured by the Rating Record of Picture Hits.

A single-subject design recommends that the baseline should be measured continually for at least three days. The trend toward stability of results at baseline is an important indicator for the decision to introduce the intervention (independent variable). The progression and, subsequently, the stability of gains or the achievement of objectives are also important indicators for the decision to conclude the intervention (Kazdin, 1982; Lourenço et al., 2009).

From this perspective, after the stability of the baseline, the intervention for the analysis of the effects of the digital memory game activity was begun. The intervention, which was mediated by the first author, was conducted over eight sessions lasting approximately $30 \mathrm{~min}$ each, performed twice per week. At the start and at the end of each phase, the necessary instructions were given for the continuance of the activity by means of oral communication. During the intervention with the game, at each phase won, the mediator gave feedback to the child with positive affirmations and, when the child exhibited difficulties in associating the cards, he was guided with examples of day-to-day situations. The effects of the intervention were measured at the end of each session by means of the Rating Record of Picture Hits. The level of agreement of observations of picture hits, performed by the first author, was tested approximately $37 \%$ of the time with two independent observers.

\section{Results \\ Usability analysis of the digital game}

Table 1 shows the data recorded by the game in relation to the number of attempts and the total time spent interacting with the activity during the usability analysis.

The results indicated an increase in the gaming time and the number of attempt in Phase 3, the phase in which the game's strategy requires greater memory skill by hiding all the cards at once. However, the child's performance during the usability analysis and her ability

Table 1 Gaming time and number of attempts recorded in the usability analysis

\begin{tabular}{lllll}
\hline Indicator & Phase 1 & Phase 2 & Phase 3 & Phase 4 \\
\hline Gaming time (min) & $6: 54$ & $3: 14$ & $7: 29$ & $6: 10$ \\
Number of attempts & 9 & 7 & 20 & 17 \\
\hline
\end{tabular}


to complete all of the phases suggests the overall viability of the use of the game by the child.

Table 2 shows the results of the other items observed during the usability analysis.

Taking into account the user's short time of familiarization with the game, the results obtained in the analysis of usability are considered satisfactory because problems related to the interface, the time available for the game, and the rules presented were not recorded. The level of agreement between the researcher and observer A was $78 \%$ and $89 \%$ with observer B, which are both considered satisfactory. The items on which the researcher and the observers disagreed were the following: the interaction with the game interface, which was recorded by the observers as regular; and the difficulty in differentiating the phases (observer A disagreed that the child had difficulty).

\section{Effectiveness of the digital memory game}

The baseline identified the need to perform the intervention with the digital game because the child did not match all of the ADL-related pictures in the paper cards correctly over the three consecutive evaluation sessions. Table 3 shows these results in detail.

The results in Table 3 show that the pairs of cards that were never matched correctly by the child during the three baseline sessions as follows: the cup with water/ drinking water, the book/reading the book, and the tissue box/blowing one's nose. The remaining pairs of cards fluctuated between hits and misses.

Based on the baseline results, an intervention was performed with the digital memory game. The effects of the intervention are displayed in Fig. 2.

As Fig. 2 shows, at the intervention stage, the results indicate some instability in the number of hits in the four initial sessions. As the intervention progressed, the number of hits and the stability increase, suggesting the influence of the game in the recognition of the pictures. The overall level of agreement between the researcher's

Table 2 Results of the usability analysis

\begin{tabular}{|c|c|}
\hline \\
\hline \multicolumn{2}{|l|}{ How was the interaction with the interface? } \\
\hline How was the understanding of the rules? & Regular \\
\hline $\begin{array}{l}\text { Did you associate and recognize the corresponding pictures } \\
\text { correctly? }\end{array}$ & Yes \\
\hline Was it difficult to differentiate the phases? & Yes \\
\hline $\begin{array}{l}\text { Did you consider the information on gaming time, phase, and } \\
\text { number of attempts? }\end{array}$ & No \\
\hline Level of difficulty encountered in phase 1 & Low \\
\hline Level of difficulty encountered in phase 2 & Low \\
\hline Level of difficulty encountered in phase 3 & Medium \\
\hline Level of difficulty encountered in phase 4 & Medium \\
\hline
\end{tabular}

Table 3 Hits and misses at baseline

\begin{tabular}{|c|c|c|c|c|c|c|}
\hline \multirow[t]{2}{*}{ Picture (pair) } & \multicolumn{2}{|c|}{ Session 1} & \multicolumn{2}{|c|}{ Session 2} & \multicolumn{2}{|c|}{ Session 3} \\
\hline & Hit & Miss & Hit & Miss & Hit & Miss \\
\hline Ball/playing ball & $\mathrm{x}$ & & $\mathrm{x}$ & & $x$ & \\
\hline Cup of water/drinking water & & $\mathrm{x}$ & & $\mathrm{x}$ & & $\mathrm{x}$ \\
\hline Plate of food/eating & $\mathrm{x}$ & & & $\mathrm{x}$ & $\mathrm{x}$ & \\
\hline Bed/making the bed & & $x$ & $\mathrm{x}$ & & $\mathrm{x}$ & \\
\hline Book/reading the book & & $x$ & & $x$ & & $x$ \\
\hline Toothbrush/brushing one's teeth & $x$ & & $\mathrm{x}$ & & & $\mathrm{x}$ \\
\hline Tissue box/blowing one's nose & & $\mathrm{x}$ & & $x$ & & $x$ \\
\hline Soap/taking a bath & $x$ & & $\mathrm{x}$ & & $\mathrm{x}$ & \\
\hline Pencil/drawing & $\mathrm{x}$ & & $\mathrm{x}$ & & & $x$ \\
\hline Shirt/putting on a shirt & $\mathrm{x}$ & & $\mathrm{x}$ & & & $\mathrm{x}$ \\
\hline
\end{tabular}

evaluation and the independent observers is satisfactory (96.7\%).

\section{Discussion}

This study seeks to evaluate the usability of a digital memory game designed for children with $\mathrm{CP}$ to address ADL and to analyze the effectiveness of this game for the association of corresponding objects and actions. With regard to usability, especially the question of accessibility and interface, the results reveal the importance of considering the needs of a specific audience during the

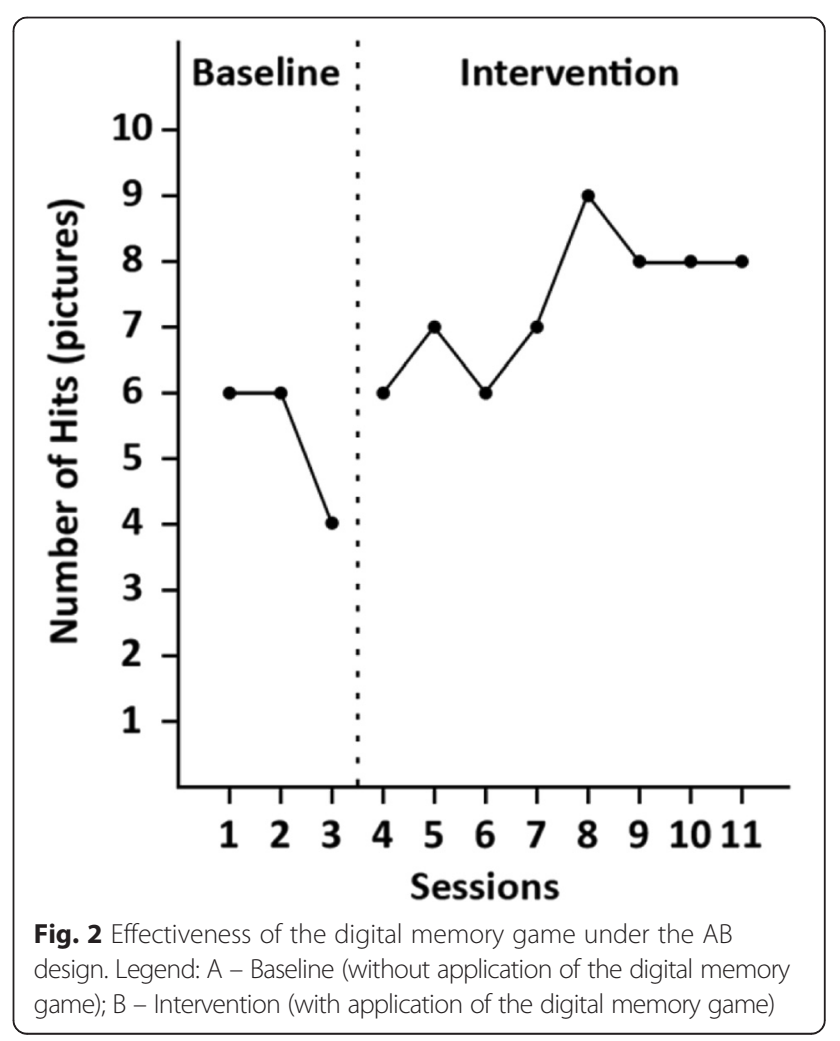


design of digital games aimed at providing a product capable of efficiently attending to its users and still achieving specific goals in learning, as recommended in the literature (Garris, Ahlers, \& Driskell, 2002; Virvou \& Katsionis, 2008). The results related to the specific difficulties in usability and in confronting challenges seem to be common to any player who is in the initial period of immersion in the activity and understanding its rules. Thus, the results reinforce the considerations of Cybis, Betiol, and Faust (2007) that the quality of an educational game is related to the analysis of the different elements of its use and of the participation of the user in this decision process of the interface, as occurred in this study.

Compared with the baseline, the results show that the performance of intervention with the digital memory game may have influenced the progress of picture matching hits because greater recognition of ADL actions was observed. This information supports the findings of authors who indicate the importance of employing digital games in learning processes, considering that, in the development of this technology, the inputs of the system should be composed of the characteristics of the game and the related educational content (Garris et al., 2002; Robertson \& Howells, 2008); care was taken to do so in this study. The fact that digital gaming technology allows intensifying the challenges and increasing the complexity of the phases, in addition to having attractants such as colors, drawings, and electronic devices, can make the person feel more challenged and motivated to play, and the repetition of the same information several times can accelerate and enhance the degree of transfer of information from short-term memory to longterm memory (Izquierdo, 2011).

Digital memory gaming involves motivation and possibly creates a pleasurable response, for example, passing a phase. The permission to pass a phase requires prior information on the game to ensure that the player does not commit errors. Pre-existing memories are accessed by working memory, which manages the player's actions. In this case, digital memory gaming, by means of the conditioned or instrumental response, can provide for associative learning that is common and of great adaptive value. Considering the temporal sequence in the storage of information, memory consolidation requires the mental repetition of this information, which may facilitate, for instance, the storage of basic information concerning everyday life and related to speaking, writing, motor activity, and other essential activities. Subsequently, it is possible that the long-term storage of this information can contribute to forming procedural memories, which involve habits, motor skills, sensory skills, and cognitive skills (Izquierdo, 2011). Therefore, an integration of various types of memories that are formed by playing memory games can contribute to greater cognitive, motor, and sensorial performance and, consequently, a higher quality of life.
The directions for using the game, given by the mediator, are important for the interaction of the child with $\mathrm{CP}$ with the digital memory game. Robertson and Howells (2008) argue that, for a game to be applied in a classroom or in other educational contexts, the effective mediation of a teacher or facilitator becomes important for learning acquisition. Technology, mediated by a facilitator, expands the procedures and the techniques employed in learning situations and can strengthen their effects, as shown in Pivik, Mccomas, Macfarlane, and Laflamme (2002). Thus, professionals should be conscientious of the importance of computational technology and electronic media in the context of contemporary learning (Connolly et al., 2012).

\section{Conclusion}

The digital game developed for this study may be considered an AT, given its specificities and the categories adopted by the Committee on Technical Assistance: aids for everyday life and practical life; augmentative and/or alternative communication (AAC); and computer accessibility resources (Brazil, 2009). The digital game for ADL tested in this study, which can be uploaded onto a PDA and used via touchscreen display and which can enhance the different forms of communication for persons with speech disorders by means of AAC boards on the computer, is an AT that may be a viable resource for children with CP.

Although the methodological limitations of this study do not invalidate its results, new studies may continue the production of knowledge in this area by means of the following: (a) increasing the sample size; (b) creating new strategies to work with digital games, for instance, group activities or activities within the family environment; and (c) broadening the research objectives and analyzing the effects of the intervention on the everyday life of the child. Additionally, future studies should create models to provide parents or those responsible for the child with $\mathrm{CP}$ to awareness of ADL practices as a means of independence, including using the digital game proposed here as a resource in the intervention.

\section{Competing interests}

The authors declare that they have no competing interests.

\section{Authors' contributions}

This paper is a result of the research conducted by SNSdC during his Masters program, under the guidance of CdSP-G. XLTJ worked on the development of the technological product. RG contributed to the development of the conceptual model of the study on memory. All authors read and approved the final manuscript.

Financial support

Bahia State Research Support Foundation (Fundação de Amparo à Pesquisa do Estado da Bahia - FAPESB). 


\section{Author details}

${ }^{1}$ Faculdade de Tecnologia SENAI CIMATEC, Salvador, Brazil. ${ }^{2}$ Universidade Federal de Santa Catarina, Joinville, Brazil. ${ }^{3}$ UNIME, Faculdade Ruy Barbosa, Salvador, Brazil.

\section{Received: 8 February 2016 Accepted: 2 March 2016}

\section{Published online: 13 April 2016}

\section{References}

Beckung E, Hagberg G. Neuroimpairments, activity limitations, and participation restrictions in children with cerebral palsy. Dev Med Child Neurol. 2002;44:309-16. doi:10.1111/j.1469-8749.2002.tb00816.x.

Brazil. Subsecretaria Nacional de Promoção dos Direitos da Pessoa com Deficiência. Comitê de Ajudas Técnicas - Tecnologia Assistiva. Brasilia: CORDE; 2009.

Brown M, Gordon WA. Impact of impairment on activity patterns of children. Arch Phys Med Rehabil. 1987;68:828-32.

Connolly TM, Boyle EA, MacArthur E, Hainey T, Boyle JM. A systematic literature review of empirical evidence on computer games and serious games. Comput Educ. 2012;59:661-86. doi:10.1016/j.compedu.2012.03.004.

Cybis WA, Betiol AH, Faust R. Ergonomia e usabilidade: Conhecimentos, métodos e aplicações. São Paulo: Novatec; 2007.

Garris R, Ahlers R, Driskell JE. Games, motivation and learning. Simulation \& Gaming. 2002;33:43-56. doi:10.1177/1046878102238607.

Guerzoni VPD, Barbosa AP, Borges ACC, Chagas PSC, Gontijo APB, Eterovick F, Mancini MC. Analysis of occupational therapy interventions in the performance of everyday activities in children with cerebral palsy: a systematic review of the literature. Rev Bras Saúde Matern Infant. 2008:8:17-25. doi:10.1590/S1519-38292008000100003.

International Organisation for Standardisation. ISO 9241-11: Ergonomic requirements for office work with visual display terminals (NDTs). Part 11 — Guidelines for specifying and measuring usability. Gènève: International Organisation for Standardisation; 1998.

Izquierdo I. Memória. Porto Alegre: Artmed; 2011.

Kazdin AE. Single-case research designs: Methods for clinical and applied settings. New York: Oxford University Press; 1982.

Kearney PR. Playing in the Sandbox: Developing games for children with disabilities. Digital Games Research Association, Conference: Changing Views - Worlds in Play. 2005.

Lourenço EAG, Hayashi MCPI, Almeida MA. Delineamentos intrassujeitos nas dissertações e teses do PPGEES/UFSCAR. Rev bras educ espec. 2009;15:319-36. doi:10.1590/S1413-65382009000200010

Matsukura TS, Marturano EM. Catálogo de avaliação do nível de independência de crianças de 4 a 8 anos nas atividades de vida diária. São Carlos: EdUFSCar; 2001.

Mutch LW, Alberma E, Hagber B, Kodam K, Perat MV. Cerebral palsy epidemiology: Where are we now and where are we going? Dev Med Child Neurol. 1992;34:547-55. doi:10.1111/j.1469-8749.1992.tb11479.x.

Pivik J, Mccomas J, Macfarlane I, Laflamme M. Using virtual reality to teach disability awareness. J Educ Comput Res. 2002;26:203-18. doi:10.2190/WACX-1VR9-HCMJ-RTKB.

Robertson J, Howells C. Computer game design: opportunities for successful learning. Comput Educ. 2008;50:559-78. doi:10.1016/j.compedu.2007.09.020.

Sampaio AAS, Azevedo FHB, Cardoso LRD, Lima C, Pereira MBR, Andery MAPA. Uma introdução aos delineamentos experimentais de sujeito único. Interação em Psicologia. 2008;12:151-64. doi:10.5380/psi.v12i1.9537.

Sternberg RJ. Psicologia Cognitiva. São Paulo: Cengage Learning; 2010.

United States. The Americans with Disabilities Act: Questions and answers. Washington, DC: Equal Employment Opportunity Commission; 2004

Virvou M, Katsionis G. On the usability and likeability of virtual reality games for education: the case of VR-ENGAGE. Comput Educ. 2008:50:154-78. doi:10.1016/j.compedu.2006.04.004

\section{Submit your manuscript to a SpringerOpen ${ }^{\circ}$ journal and benefit from:}

- Convenient online submission

- Rigorous peer review

Immediate publication on acceptance

- Open access: articles freely available online

- High visibility within the field

- Retaining the copyright to your article 